\title{
Timing of surgery following neoadjuvant chemoradiotherapy in locally advanced rectal cancer - A comparison of magnetic resonance imaging at two time points and histopathological responses
}

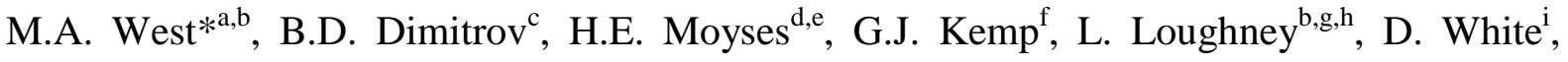

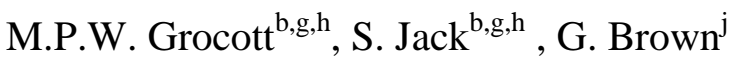

${ }^{a}$ Academic Unit of Cancer Sciences, Faculty of Medicine, University of Southampton, Southampton, United Kingdom

${ }^{\mathrm{b}}$ Integrative Physiology and Critical Illness Group, Clinical and Experimental Sciences, Faculty of Medicine, University of Southampton, University Road, Southampton, United Kingdom

${ }^{c}$ Primary Care and Population Sciences, University of Southampton, Southampton, United Kingdom

d National Institute for Health Research, Southampton Biomedical Research Centre, University Hospital Southampton NHS Foundation Trust and University of Southampton, Southampton, UK

${ }^{\mathrm{e}}$ National Institute for Health Research, Southampton Respiratory Biomedical Research Unit, University Hospital Southampton NHS Foundation Trust and University of Southampton, Southampton, UK

${ }^{\mathrm{f}}$ Department of Musculoskeletal Biology, Faculty of Health and Life Sciences, University of Liverpool, Liverpool, United Kingdom

${ }^{\mathrm{g}}$ Critical Care Research Area, Southampton NIHR Respiratory Biomedical Research Unit, Southampton, United Kingdom

${ }^{\mathrm{h}}$ Anaesthesia and Critical Care Research Unit, University Hospital Southampton NHS Foundation Trust, Southampton, United Kingdom

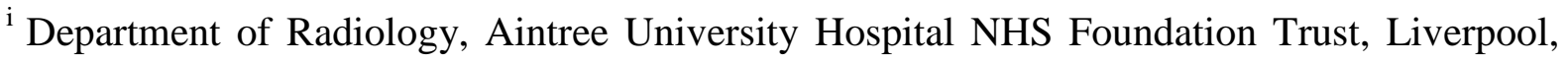


UK

${ }^{\mathrm{j}}$ Department of Radiology, The Royal Marsden NHS Foundation Trust, London, UK

Authors email addresses:

MAW m.west@soton.ac.uk

BDD $\underline{\text { b.dimitrov@ } \text { soton.ac.uk }}$

HM h.e.moyses@ soton.ac.uk

GJK g.j.kemp@liverpool.ac.uk

LL1.loughney@gmail.com

DW david.white@aintree.nhs.uk

MPWG mike.grocott@ soton.ac.uk.

SJ $\underline{\text { s.jack@ @oton.ac.uk }}$

GB gina.brown@rmh.nhs.uk

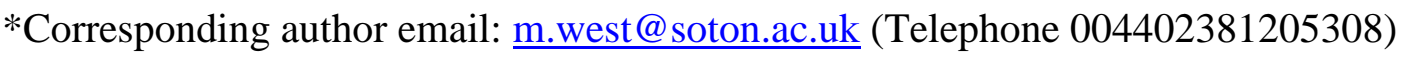

Mr Malcolm West

Academic Unit of Cancer Sciences,

Faculty of Medicine,

University Surgery, University Hospital Southampton

Tremona Road

Southampton,

United Kingdom

Short title - Association between MRI staging and histopathology 


\section{ABSTRACT}

Purpose: There is wide inter-institutional variation in the interval between neoadjuvant chemoradiotherapy (NACRT) and surgery for locally advanced rectal cancer. We aimed to assess the association of magnetic resonance imaging (MRI) at 9 and 14 weeks post-NACRT; T-staging (ymrT) and post-NACRT tumour regression grading (ymrTRG) with histopathological outcomes; histopathological T-stage (ypT) and histopathological tumour regression grading (ypTRG) in order to inform decision-making about timing of surgery.

Patients and Methods: We prospectively studied 35 consecutive patients (26 males) with MRI-defined resection margin threatened rectal cancer who had completed standardized NACRT. Patients underwent a MRI at Weeks 9 and 14 post-NACRT, and surgery at Week 15. Two readers independently assessed MRIs for ymrT, ymrTRG and volume change. ymrT and ymrTRG were analysed against histopathological ypT and ypTRG as predictors by logistic regression modelling and receiver operating characteristic (ROC) curve analyses.

Results: Thirty-five patients were recruited. Inter-observer agreement was good for all MR variables (Kappa>0.61). Considering ypT as an outcome variable, a stronger association of favourable ymrTRG and volume change at Week 14 compared to Week 9 was found (ymrTRG $-\mathrm{p}=0.064$ vs. $\mathrm{p}=0.010 ;$ Volume change $-\mathrm{p}=0.062$ vs. $\mathrm{p}=0.007$ ). Similarly, considering ypTRG as an outcome variable, a greater association of favourable ymrTRG and volume change at Week 14 compared to Week 9 was found (ymrTRG $-p=0.005$ vs. $p=0.042$; Volume change $-\mathrm{p}=0.004$ vs. 0.055$)$.

Conclusion: Following NACRT, greater tumour downstaging and volume reduction was observed at Week 14. Timing of surgery, in relation to NACRT, merits further investigation. 


\section{Keywords}

Magnetic resonance imaging, surgery, rectal cancer, tumour regression, time for surgery

\section{Trial Registration Number}

NCT: 01325909 


\section{INTRODUCTION}

In the UK colorectal cancer is the third commonest cause of cancer death [1,2] and 5000 patients underwent surgery for rectal cancer ( $71 \%$ aged $>65$ years) during 2014 . In $25 \%$ of these patients, major resection was preceded by neoadjuvant chemoradiotherapy (NACRT) [3], with the aim of controlling local disease and achieving tumour downsizing and negative resection margins, with marginal gains in overall survival [4-8]. High-resolution pelvic magnetic resonance imaging (MRI) is now the gold-standard in preoperative rectal cancer staging [9]. The decision to administer NACRT is based on identifying MRI-defined circumferential resection margin (CRM) threatened cancers.

Histopathologists grade tumour response in three ways: firstly assessment of the status of the CRM, secondly the depth of tumor spread and nodal status (ypT and ypN stage), and thirdly by evaluating tumor regression grade (ypTRG) [10,11]. A number of studies have shown that both ypT and ypN stage are independent predictors of outcome, and several retrospective studies report a link between outcome and histopathology assessment of final stage or tumor regression after NACRT $[12,13]$. Accurate preoperative assessment of response to therapy may permit the clinical teams to modify definitive treatment [14]. A number of different methods have been proposed for assessing response of rectal cancer to CRT on MRI. These include post-treatment $\mathrm{T}$ staging (ymrT), volume reduction between baseline and posttreatment, [15] and modified Response Evaluation Criteria in Solid Tumors (RECIST) measurement [16]. In addition to these assessment criteria, the MERCURY study group has developed an MRI-based tumor regression grading (ymrTRG) system by applying the principles of histopathology ypTRG $[17,18]$ and showed that MRI assessment of ypTRG following preoperative therapy predicted survival [17]. It has been suggested that there may 
be benefits in prolonging the interval between end of NACRT and surgery beyond the common 6-8 weeks [19-21], but evidence is limited.

The aim of this study was to assess MRI-defined favourable versus unfavourable responders (ymrT, ymrTRG and change in volume) at two time-points post-NACRT and to compare these evaluations with histopathological ypT and ypTRG, in an attempt to inform decisions about optimal timing of surgery with respect to NACRT. We also explored the level of interobserver agreements between central and local MR reviewers for ymrT, mrTRG and volume change at both time points. 


\section{PATIENTS AND METHODS}

\section{Patients and Study Design}

This prospective pilot trial was performed as a nested sub-study within a larger trial [22] approved by the North West - Liverpool East Research and Ethics Committee (11/H1002/12) and registered with ClinicalTrials.gov (NCT01325909). Written informed consent was obtained from all patients. We recruited consecutive patients between August 2012 and August 2014 referred to the Colorectal Multi-Disciplinary Team (MDT), age $\geq 18$ years, with locally advanced (circumferential resection margin threatened - defined as tumour within 2 $\mathrm{mm}$ of the mesorectal fascia or if any $\mathrm{T} 3 / 4$ tumour was arising at $<5 \mathrm{~cm}$ from the anal verge) resectable rectal cancer, scheduled for standardized NACRT on the basis of Tumour, Node, Metastasis (TNM) classification $>\mathrm{T} 2 / \mathrm{N}+$ with no distant metastasis [23] and WHO Performance Status < 2 [24]. Exclusion criteria were: inability to give informed consent, nonresectable disease, and patients who declined surgery or NACRT, or who received nonstandard NACRT.

All patients underwent TNM staging involving flexible sigmoidoscopy to obtain tissue for histological diagnosis, completion colonoscopy, chest, abdomen and pelvis computer-aided tomography (CT) and 1.5 Tesla pelvic magnetic resonance imaging (MRI) at baseline. All patients completed 5 weeks NACRT. Standardized radiotherapy consisted of 45 Gy in 25 fractions on weekdays using a 3D conformal technique with CT guidance. A boost dose was given (5.4 Gy in 3 fractions) to the primary tumour only. Oral capecitabine $\left(825 \mathrm{mg} . \mathrm{m}^{-2}\right.$ ) was given twice daily on radiotherapy days. No patient received brachytherapy. At 9 weeks postNACRT, patients were restaged using chest, abdomen and pelvic CT and pelvic MRI. At 14 weeks post-NACRT, patients were restaged using pelvic MRI, prior to surgery at Week 15 . 


\section{MRI Technique}

MRI technique was performed as described by Patel and colleagues [25,26]. MR image analysis was carried out, using the terms ymrT ( $\mathrm{T}$ stage on MRI images obtained after NACRT), ymrTRG (tumor regression grade on MRI images obtained after NACRT), ypT (T stage on post-treatment histopathological examination of the resection specimen), and ypTRG (tumor regression grade on post-treatment histopathological examination of the resection specimen) to describe the data [25,27]. The MRI scans were anonymised and separately reviewed by two radiologists (Central reviewer; GB and Local reviewer; DW) with 20 and 15 respective years of experience in MRI assessment of rectal cancers, in two tertiary referral colorectal cancer centres, using previously defined criteria.

\section{MRI Image Analyses}

Images were analysed for ymrTRG, ymrT and percentage volume change. ymrT was based on the interpretation of local extent of persistent tumor signal intensity relative to the layers of bowel wall on T2-weighted images. Comparison was made with the pre-treatment images. Tumour response manifested as either replacement of tumor signal by low signal intensity fibrosis (dark stroma) or the development of high signal intensity mucin pools; such areas were not considered to be tumour, as they did not contribute to $\mathrm{T}$ staging. ymrT staging is was conducted as described by Sobin and Brierley [23,28]. T3 sub-staging was conducted as described by Patel and colleagues [25].

Based on known histopathological outcomes according to ypT stage, the patient's ymrT was divided into favourable and unfavourable response to enable binary comparison. Favourable was defined as stages ymrT0, 1, 2, and 3a, while unfavourable was defined as ymrT3b, c, d and ymrT4 [29]. 
MRI TRG is based on principles similar to the pathological ypTRG system originally described by Dworak subsequently modified using the Mandard scale. Scans were reviewed to determine the degree of tumor replacement by fibrotic stroma, as previously described $[17,18,26]$. Favourable MRI tumor regression grade was defined as grades 1, 2, and 3, and unfavourable regression as grades 4 and 5 , as in previous studies [17,25].

The MRI scans were also assessed for percentage volume change [25]. Tumor volume was obtained by multiplying tumor length, width, and height. Percentage volume reduction was defined as $100 \times\{($ Volume at baseline $)-($ Volume post-CRT $)\} /($ Volume at baseline $)$, and categorized into two groups (unfavourable $<80 \%$, and favourable $\geq 80 \%$ ) using previously published definitions [15].

\section{Surgical Resection}

All patients underwent total mesorectal excision (TME) [30] with or without abdominoperineal excision, performed 15 weeks (+/- 4 days) after the completion of NACRT.

\section{Histopathology Assessment}

After surgical resection, the specimen was fixed in formalin for $48 \mathrm{~h}$, cross-sectioned into 3-5 mm slices, and histologically sampled (MT - acknowledged). A predefined protocol assessed pathological complete response, with a minimum of 5 blocks of tumour taken. If no tumour was found on the first set of haematoxylin and eosin sections the rest of the tumor area was embedded, and if no tumour was seen then a final three levels were taken through each block to look for tumor to confirm a complete response. Each specimen was graded by degree of tumour regression, according to the Dworak system and also by ypT stage. As well as grading and staging by the five-point ypTRG and TNM version 5 systems, a simplified 
pathological grading of favourable and unfavourable pathology was also undertaken. Favourable pathology was defined as ypT stages $0,1,2$, and 3a or ypTRG stages 3 and 4 . Unfavourable pathology was defined as ypT stages $3 b, c, d$, and 4 or ypTRG stages 0,1 , and 2. ypT3a was included in the favourable group as these tumours have been shown to have a similar prognostic outcome as ypT2 tumours $[18,29]$.

\section{Statistical Analysis}

Central reviewer (Royal Marsden; GB) data was used for the primary analysis; agreement between the two observers grading categorical variables (ymrT, ymrTRG and volume change) was determined by kappa statistic $(\kappa=0$, poor agreement; $\kappa=0-0.20$, slight agreement; $\kappa=0.21-0.40$, fair agreement; $\kappa=0.41-0.60$, moderate agreement; $\kappa=0.61-$ 0.80 , substantial agreement; and $\kappa=0.81-1.00$, almost perfect agreement).

Data were described as frequency (percentage) and mean (SD), with 95\% confidence intervals (95\% Cis), as appropriate. To analyse the association between demographic variables (age and sex), MRI parameters (ymrT, ymrTRG, volume change) and pathologic tumor response (ypT and ypTRG), univariate logistic regression analysis or Fischer's exact test was used. Logistic regression enabled calculation of odds ratio (OR) along with 95\%CIs where possible. Receiver operating characteristic (ROC) curve analysis was also performed, with calculation of the area under the curve (AUC) as an indicator of overall accuracy, together with sensitivity, specificity, positive and negative likelihood ratios. Significant univariate relationships with the outcome were adjusted by multifactor logistic regression analysis for baseline values of the predictor variables if this was possible. Two-tailed $\mathrm{p}<0.05$ was considered statistically significant unless specified otherwise. Calculations were performed using Statistical Package for Social Sciences program, version 22.0 (SPSS, IBM, USA) and Stata, version 11.2 (StataCorp. College Station, TX) 


\section{RESULTS}

Table 1 shows the baseline demographic characteristics of the patients who were eligible for this study. All patients completed the standardised course of NACRT. One patient needed capecitabine dose reduction, while 4 patients sustained perineal radiation skin changes (maximum score 2 out of 4 ).

Table 2 shows MRI T-stage, TRG, volume change at Week 9 and Week 14 and pathological T-stage and TRG. The mean baseline distance from anal verge was $54 \mathrm{~mm}$, standard deviation (SD) $28 \mathrm{~mm}$. The mean baseline tumour length was $50 \mathrm{~mm}$ (SD $18 \mathrm{~mm}$ ). The mean baseline tumor volume was $47773 \mathrm{~mm}^{3}$ (SD $72005 \mathrm{~mm}^{3}$ ). The mean post-treatment tumor distance from anal verge at Week 9 was 56 mm (SD 27mm), and at Week 14 was 66 mm (SD $28 \mathrm{~mm}$ ). The mean Week 9 tumour volume was $16277 \mathrm{~mm}^{3}\left(\mathrm{SD} 29386 \mathrm{~mm}^{3}\right.$ ) and at Week 14 was $8831 \mathrm{~mm}^{3}\left(\mathrm{SD} 18060 \mathrm{~mm}^{3}\right)$. The mean tumor volume reduction at Week 9 was $61 \%$ (SD $39 \%$ ) and at Week 14 was $80 \%$ (SD 22\%). At histopathological examination the mean number of blocks taken was 7 (SD 4) and the mean number of sections taken per block was 7 (SD 4). The mean nodal harvest was 12 (SD 8). Poor quality MR images $(n=5)$ and missing pathological staging $(n=2)$ data were reported in the Table 1 and 2 for completeness however these data were not used in the generation of logistic regression models.

\section{Interobserver Agreement}

Appendix 1 shows the raw data for the interobserver agreements between central and local MR reviewers for ymrT, ymrTRG and volume change. Agreements ranged between moderate $(\kappa=0.44)$ to almost perfect $(\kappa=0.92)$ for continuous and categorical variables at all 3 time points (baseline, Week 9 and Week 14). Importantly, ymrTRG agreements at Week 9 and 14 are almost perfect between the two reviewers. 
T stage, tumour regression grading and volume change at Week 9 and Week 14 on MRI images obtained after NACRT

Table 3 shows univariate logistical regression models of age, gender, ymrT, ymrTRG and volume change at Week 9 and Week 14 compared to ypT histopathology grade. Tumour grading of ymrT stage T0-T3a was significantly associated with favourable pathology at Week 14, compared with ymrT stage T3b-4 $(\mathrm{p}=0.006)$. ymrT at Week $14(\mathrm{p}=0.003)$ and at Week $9(\mathrm{p}=0.002)$ only showed a tendency for difference when related to favourable pathology. Furthermore, tumours graded as ymrTRG stage 1-3 were significantly associated with favourable pathology at Week 14 , compared with ymrTRG stage $4-5(\mathrm{p}=0.009)$. In particular, Week 14 showed a stronger association $(\mathrm{p}=0.01)$ of ymrTRG and favourable pathology than Week $9(\mathrm{p}=0.064)$. These models were not amenable to adjustment for baseline values.

Tumours graded as favourable volume change $(\geq 80 \%)$ were significantly associated with favourable pathology at Week $14(\mathrm{p}=0.007)$. Week 14 showed a stronger association of favourable volume change when compared to favourable ypT stage than volume change at Week $9(\mathrm{p}=0.062)$. Moreover, when adjusting for baseline values, volume change at Week 14 was still significantly associated with favourable ypT stage $(\mathrm{p}=0.025)$. The sensitivity, specificity, positive and negative likelihood ratios for all of the variables described above are plotted in Figure 1 and tabulated in Appendix 2. Higher sensitivity, specificity and respective area under the receiver operating characteristic (AUC) curve measurements were received for Week 14 measurements.

Table 4 shows a similar univariate logistical regression model of age, gender, ymrT and ymrTRG at Week 9 and Week 14 compared to ypTRG derived at histopathology. Again, ymrT stage T0-T3a is significantly associated with favourable pathology at Week 14, 
compared with ymrT stage T3b-4 $(\mathrm{p}=0.017)$. ymrT at Week $14(\mathrm{p}=0.011)$ and at Week 9 $(\mathrm{p}=0.020)$ only showed a tendency for difference when related to favourable pathology. However, after adjusting for baseline values, ymrT at Week 14 showed more association with favourable ypTRG stage $(\mathrm{p}=0.035)$ that ymrT at Week 9.

Tumours graded as ymrTRG stage 1-3 were significantly associated with favourable pathology at Week 14, compared with ymrTRG stage 4-5 ( $\mathrm{p}=0.005)$. This showed a stronger association with favourable pathological outcome than ymrTRG at Week $9(\mathrm{p}=0.042)$. These models were not amenable to adjustment for baseline values.

Similarly, tumours graded as favourable volume change $(\geq 80 \%)$ were significantly associated with favourable pathology at Week $14(\mathrm{p}=0.004)$. Week 14 showed a stronger association of favourable volume change when compared to favourable ypTRG stage than volume change at Week $9(\mathrm{p}=0.055)$. When adjusting for baseline values, volume change at Week 14 became more significantly associated with favourable ypTRG stage $(\mathrm{p}=0.015)$. The sensitivity, specificity, positive and negative likelihood ratios for all of the variables described above are plotted in Figure 1 and tabulated in Appendix 2. Higher sensitivity, specificity and respective area under the receiver operating characteristic (AUC) curve measurements were received for Week 14 measurements. 


\section{DISCUSSION}

In this study we have compared MRI evaluation of ymrT-staging, ypTRG and tumour volume assessments at two time-points (Week 9 and Week 14) post-NACRT with the pathology gold standards of ypT and ypTRG. This is the first attempt at understanding the relationship between MRI derived predictors and histopathological outcomes at two time points postNACRT prior to surgery, in an attempt to inform clinical decision making about the optimal time interval between the end of NACRT and surgery. This is the first prospective study of tumour changes on MR at two pre-operative restaging time points, and the first report of substantial agreement for ymrT and tumour volume at baseline, Week 9 and Week 14 and most importantly, the first report of almost perfect agreement on ymrTRG at Week 9 and Week 14 between two blinded reviewers at two different tertiary colorectal cancer referral centres.

Considering ypT as an outcome variable, there was a stronger association of favourable ymrTRG and volume changes at Week 14 compared to Week 9. Similarly, when considering ypTRG as an outcome variable, there was a stronger association of favourable ymrTRG and volume changes at Week 14 compared to Week 9. All predictor variables at Week 14 show a strong relationship with both histopathological parameters. Clearly tumour regression is still ongoing up until Week 14 post-NACRT. A greater mean tumor volume reduction at Week 14 $(80 \%($ SD 22\%)) than at Week $9(61 \%$ (SD 39\%)) was also shown confirming a strong association between favourable tumour volume changes at Week 14 compared to Week 9 for both outcome variables. Interestingly, after correcting the volume change regression models for baseline values, the Week 14 models were still significantly related to both histopathological outcome measures, unlike the Week 9 models. The Week 14 predictive models corrected for baseline still retain their significant association with outcome variables, 
and are indicative of optimal variables that can be used in clinical practice; however larger validation studies are necessary to confirm this finding further.

The pre-operative MRI staging of locally advanced, circumferential margin-threatened rectal cancers is closely associated with survival outcomes. MRI assessment post-NACRT has implications for surgical planning, timing of surgery, sphincter preservation and (for favourable responders) perhaps the deferral of surgery. Thus the ability to use re-staging MRI variables like ypT, ypTRG and volume change to predict favourable and unfavourable pathological outcomes in a clinical setting is crucial as the subgroup of patients with MRpredicted unfavourable outcomes are at a higher risk of local or systemic failure following oncological resection. In these cases pre-operative MR may not only direct surgical dissection, but also alert the MDT to the need for further upfront systemic chemotherapy, contact radiotherapy or extended surgical resection. In this cohort of patients, the identification of an optimal time for surgery post-NACRT which coincides with maximal oncological down-staging is an urgent question [21,31]. This pilot study suggests that further volume reduction and down-staging occurs between Week 9 and Week 14 post-NACRT, with more favourable ymrT, ymrTRG and volume changes found at Week 14. Moreover, the longer time to surgery post-NACRT was associated with a $23 \%$ pathological complete response rate (pCR), a high rate comparable to literature rates of $17-27 \%$ [32]. These results lend support to previous work where a greater delay to surgery following completion of NACRT is associated with better pathological outcome $[19,25]$. The link between greater down-staging, completed pathological responses and long-term impact on disease-free survival are however yet to be established. This highlights the need for a randomised controlled trial. 
The main limitations of this study were (i) the limited number of patients recruited in what was a nested study within a larger published trial. We suggest that this potential weakness is offset by the novelty of the study design, the serial MRI assessment, the strength of the association between MRI predictor variables and histopathological outcomes and the magnitude of change between weeks 9 and 14. Other potential limitations include: (ii) the lack of tumour outlining on a workstation to calculate volume, however tumour volume calculation has been done according to a validated technique [15]; (iii) the lack of MRI nodal status reporting, however this has not been shown to be a prognostic indictor when compared with ymrT and ymrTRG [17] and (iv) the recruitment of patients from a single centre, with uncertain generalizability. Further, diffusion weighted imaging (DWI) analyses was not undertaken. To date there is no evidence that DWI is able to predict survival, unlike ymrTRG that has been validated in predicting survival [17]. Further, unlike DWI, ymrTRG does not require extra scanning time, personnel, or added investment to undertake.

Strengths of our study include: (i) the prospective study design; (ii) the homogenous study population (only operable MRI defined locally advanced rectal cancer patients); (iii) the blinded two-centre reporting of predictor variables (blind to patient demographics, clinical status, centre and timeline); (iv) the standardized NACRT regime; (v) the targeted MRI and pathological analyses, conducted by strictly following a specific protocol; and (vi) rigorous statistical modelling showing significant ORs (even after adjustment for baseline values) with calculation of predictive performance descriptors including accuracy, sensitivity, specificity, positive and negative likelihood ratios values.

Future studies of more patients may be able to refine our current findings in order to better inform decisions about optimal timing for surgical intervention following neoadjuvant cancer therapies in this cohort of patients. This will enable optimal timing of oncological resection 
based on objective, validated MRI defined tumour assessments. We suggest that clinical MRI directed re-staging based on ymrT, ymrTRG and percentage volume change would be a valuable adjunct in informing multi-disciplinary patient tailored decision-making.

\section{Acknowledgments}

Aintree University Hospitals NHS Foundation Trust, Liverpool, Colorectal MultiDisciplinary Team. Special thanks go to Dr Monica Terlizzo, Consultant Histopathologist, for histopathological analysis.

\section{Conflict of Interest}

$M P W G$ - has received research grants from: National Institute of Health Research, Medical Research Council, National Institute of Academic Anaesthesia (British Oxygen Company Chair of the Royal College of Anaesthetists), and the British Lung Foundation. He leads the Xtreme-Everest Oxygen Research Consortium, which has received un-restricted research grant funding from: BOC Medical (Linde Group) Ely-Lilly Critical Care, Smiths Medical, Deltex Medical, London Clinic, Rolex.

GB - Supported by the Royal Marsden Cancer Charity and the NIHR Biomedical Research Centre

All other authors have no declarations

\section{Role of the Funding Source}

This work was undertaken whilst MW and MPWG were funded by the National Institute of Health Research and the Royal College of Anaesthetists British Oxygen Company Fellowship awarded by the National Institute of Academic Anaesthesia for the Fit-4-Surgery 
programme of research. Funders and study sponsors had no role in the study design, in the collection, analysis and interpretation of data; in the writing of the manuscript; and in the decision to submit the manuscript for publication.

\section{References}

1. Office for National Statistics, Cancer Registration Statistics, England, 2012. http://www.ons.gov.uk/ons/rel/vsob1/cancer-statistics-registrations--england--seriesmb1-/no--42--2011/stb-cancer-statistics-registrations-2011.html (Accessed April 2015)

2. Office for National Statistics, Mortality Statistics: Colorectal Cancer, England, 2014 http://www.ons.gov.uk/ons/rel/cancer-unit/bowel-cancer-in-england/2009/sum

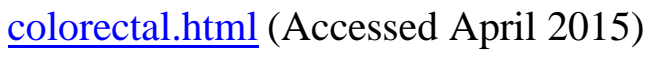

3. National Bowel Cancer Audit Report. Annual Report 2014 (accessed April 2013)

4. Habr-Gama A, Perez RO, Nadalin W, Sabbaga J, Ribeiro U, Silva e Sousa AH, Campos FG, Kiss DR, Gama-Rodrigues J. Operative Versus Nonoperative Treatment for Stage 0 Distal Rectal Cancer Following Chemoradiation Therapy. Ann Surg 2004;240(4):711-718.

5. Chau I, Brown G, Cunningham D, Tait D, Wotherspoon A, Norman AR, Tebbutt N, Hill M, Ross PJ, Massey A, Oates J. Neoadjuvant capecitabine and oxaliplatin followed by synchronous chemoradiation and total mesorectal excision in magnetic resonance imaging-defined poor-risk rectal cancer. J Clin Oncol 2006;24(4):668-674.

6. Mohiuddin M, Winter K, Mitchell E, Hanna N, Yuen A, Nichols C, Shane R, Hayostek C, Willett C. Randomized phase II study of neoadjuvant combined-modality chemoradiation for distal rectal cancer: Radiation Therapy Oncology Group Trial 0012. 
J Clin Oncol 2006;24(4):650-655.

7. Bosset J-F, Collette L, Calais G, Mineur L, Maingon P, Radosevic-Jelic L, Daban A, Bardet E, Beny A, Ollier J-C for the EORTC Radiotherapy Group Trial 22921. Chemotherapy with preoperative radiotherapy in rectal cancer. $N$ Engl $\mathrm{J}$ Med 2006;355(11):1114-1123.

8. Gérard J-P, Conroy T, Bonnetain F, Bouché O, Chapet O, Closon-Dejardin M-T, Untereiner M, Leduc B, Francois E, Maurel J, Seitz J-F, Buecher B, Mackiewicz R, Ducreux M, Bedenne L. Preoperative radiotherapy with or without concurrent fluorouracil and leucovorin in T3-4 rectal cancers: results of FFCD 9203. J Clin Oncol 2006;24(28):4620-4625.

9. Brown $\mathrm{G}$ for the Mercury Study Group. Extramural depth of tumor invasion at thinsection MR in patients with rectal cancer: results of the MERCURY study. Radiology 2007;243(1):132-139.

10. Sauer R, Becker H, Hohenberger W, Rödel C, Wittekind C, Fietkau R, Martus P, Tschmelitsch J, Hager E, Hess CF, Karstens J-H, Liersch T, Schmidberger H, Raab R for the German Rectal Cancer Study Group. Preoperative versus postoperative chemoradiotherapy for rectal cancer. N Engl J Med 2004;351(17):1731-1740.

11. Suárez J, Vera R, Balén E, Gómez M, Arias F, Lera JM, Herrera J, Zazpe C. Pathologic response assessed by Mandard grade is a better prognostic factor than down staging for disease-free survival after preoperative radiochemotherapy for advanced rectal cancer. Color Dis 2008;10(6):563-568.

12. Chan AKP, Wong A, Jenken D, Heine J, Buie D, Johnson D. Posttreatment TNM staging is a prognostic indicator of survival and recurrence in tethered or fixed rectal 
carcinoma after preoperative chemotherapy and radiotherapy. Int J Radiat Oncol Biol Phys 2005;61(3):665-677.

13. Kuo L-J, Liu M-C, Jian JJ-M, Horng C-F, Cheng T-I, Chen C-M, Fang W-T, Chung YL. Is final TNM staging a predictor for survival in locally advanced rectal cancer after preoperative chemoradiation therapy? Ann Surg Oncol 2007;14(10):2766-2772.

14. Battersby NJ, Moran B, Yu S, Tekkis P, Brown G. MR imaging for rectal cancer: the role in staging the primary and response to neoadjuvant therapy. Expert Rev Gastroenterol Hepatol 2014 Aug;8(6):703-719.

15. Yeo S-G, Kim DY, Kim TH, Jung KH, Hong YS, Chang HJ. Tumor volume reduction rate measured by magnetic resonance volumetry correlated with pathologic tumor response of preoperative chemoradiotherapy for rectal cancer. Int J Radiat Oncol Biol Phys 2010;78(1):164-171.

16. Chen CC, Lee RC, Lin JK, Wang LW, Yang SH. How accurate is magnetic resonance imaging in restaging rectal cancer in patients receiving preoperative combined chemoradiotherapy? Dis Colon Rectum 2005;48(4):722-728.

17. Patel UB, Taylor F, Blomqvist L, George C, Evans H, Tekkis P, Quirke P, SebagMontefiore D, Moran B, Heald R, Guthrie A, Bees N, Swift I, Pennert K, Brown G. Magnetic resonance imaging-detected tumor response for locally advanced rectal cancer predicts survival outcomes: MERCURY experience. $J$ Clin Oncol 2011;29(28):3753-3760.

18. Dworak O, Keilholz L, Hoffmann A. Pathological features of rectal cancer after preoperative radiochemotherapy. Int J Colorectal Dis 1997;12(1):19-23. 
19. Evans J, Tait D, Swift I, Pennert K, Tekkis P, Wotherspoon A, Chau I, Cunningham D, Brown G. Timing of Surgery Following Preoperative Therapy in Rectal Cancer: The Need for a Prospective. Dis Colon Rectum 2011;10(10):1251-1259.

20. Petrelli F, Sgroi G, Sarti E, Barni S. Increasing the Interval Between Neoadjuvant Chemoradiotherapy and Surgery in Rectal Cancer: A Meta-Analysis of Published Studies. Ann Surg 2013 Nov 20 (Epub ahead of print).

21. Foster JD, Jones EL, Falk S, Cooper EJ, Francis NK. Timing of surgery after longcourse neoadjuvant chemoradiotherapy for rectal cancer: a systematic review of the literature. Dis Colon Rectum 2013;56(7):921-30.

22. West M A, Loughney L, Lythgoe D, Barben CP, Sripadam R, Kemp GJ, MPW Grocott, Jack S. Effect of prehabilitation on objectively measured physical fitness after neoadjuvant treatment in preoperative rectal cancer patients: a blinded interventional pilot study. Br J Anaesth 2015;114(2):244-51.

23. Sobin LH, Fleming ID. TNM Classification of Malignant Tumors, Fifth Edition (1997). Cancer 1997;80(9):1803-4.

24. Oken MM, Creech RH, Tormey DC, Horton J, Davis TE, McFadden ET, Carbone PP. Toxicity and response criteria of the Eastern Cooperative Oncology Group. Am J Clin Oncol 1982;5(6):649-655.

25. Patel UB, Brown G, Rutten H, West N, Sebag-Montefiore D, Glynne-Jones R, Rullier E, Peeters M, van Cutsem E, Ricci S, Van de Velde C, Kjell P, Quirke P. Comparison of Magnetic Resonance Imaging and Histopathological Response to Chemoradiotherapy in Locally Advanced Rectal Cancer. Ann Surg Oncol 2012;19(9):2842-52. 
26. Patel UB, Blomqvist LK, Taylor F, George C, Guthrie A, Bees N, Brown G. MRI after treatment of locally advanced rectal cancer: how to report tumor response--the MERCURY experience. Am J Roentgenol. 2012;199(4):486-495.

27. Moran B, Brown G, Cunningham D, Daniels I, Heald R, Quirke P, Sebag-Montefiore D. Clarifying the TNM staging of rectal cancer in the context of modern imaging and neo-adjuvant treatment: "y" "u" and "p" need "mr" and "ct." Color Dis 2008;10(3):242-3.

28. Brierley JD, Greene FL, Sobin LH, Wittekind C. The "y" symbol: an important classification tool for neoadjuvant cancer treatment. Cancer 2006;106(11):2526-7.

29. Willett CG, Badizadegan K, Ancukiewicz M, Shellito PC. Prognostic factors in stage T3N0 rectal cancer: do all patients require postoperative pelvic irradiation and chemotherapy? Dis Colon Rectum 1999;42(2):167-73.

30. MacFarlane JK, Ryall RDH, Heald RJ. Mesorectal excision for rectal cancer. Lancet $1993 ; 341(8843): 457-60$.

31. Glimelius B. Optimal Time Intervals between Pre-Operative Radiotherapy or Chemoradiotherapy and Surgery in Rectal Cancer? Front Oncol 2014;4:50

32. Maas M, Nelemans PJ, Valentini V, Das P, Rödel C, Kuo L-J, Calvo, Felipe A, GarcíaAguilar J, Glynne-Jones R, Haustermans K, Mohiuddin M, Pucciarelli S, Small W, Suárez J, Theodoropoulos G, Biondo S, Beets-Tan R, Beets GL. Long-term outcome in patients with a pathological complete response after chemoradiation for rectal cancer: a pooled analysis of individual patient data. Lancet Oncol 2010;11(9):835-844. 
Table 1: Baseline patient characteristics

\begin{tabular}{ll}
\hline Characteristics & Values* \\
\hline Age (years) & $66.20(10.10)$ \\
Sex [male] & $26(74.30)$ \\
Height of primary tumour (from anal verge) & \\
Low $(0-5 \mathrm{~cm})$ & $18(51.31)$ \\
Medium/high $(>5 \mathrm{~cm})$ & $17(48.69)$ \\
& \\
Operation type & \\
TME & $23(65.71)$ \\
APR & $10(28.57)$ \\
Palliative & $2(5.71)$ \\
Tumour stage at baseline on MR & \\
T2 & \\
T3a & $8(22.86)$ \\
T3b & $6(17.14)$ \\
T3c & $4(11.43)$ \\
T3d & $4(11.43)$ \\
T4a & $2(5.71)$ \\
T4b & $5(14.29)$ \\
Poor quality image & $1(2.86)$ \\
& $5(14.29)$ \\
\hline
\end{tabular}

$* \mathrm{~N}=35$ patients; values are mean $(\mathrm{SD})$ or $\mathrm{n}(\%)$ 
Table 2 Patient characteristics with full MR imaging and histopathology

\begin{tabular}{lc}
\hline Characteristics & Values* \\
\hline
\end{tabular}

\section{MRI tumour T-stage post NACRT (ymrT)}

\section{Week 9}

T0

$\mathrm{T} 1$

$4(11.43)$

$\mathrm{T} 2$

$1(2.85)$

T3a

$10(28.57)$

$\mathrm{T} 3 \mathrm{~b}$

$2(5.71)$

T3c

4 (11.43)

T3d

$3(8.57)$

$1(2.86)$

T4a

5 (14.29)

Poor quality image

5 (14.29)

\section{Week 14}

T0

7 (20.00)

$\mathrm{T} 1$

5 (14.29)

$\mathrm{T} 2$

4 (11.43)

T3a

5 (14.29)

$\mathrm{T} 3 \mathrm{~b}$

4 (11.43)

T3c

1 (2.86)

T3d

$1(2.86)$

T4a

3 (8.57)

Poor quality image

5 (14.29)

\section{MRI Tumour regression grading (ymrTRG)}

\section{Week 9}

1

2

$3(8.57)$

$10(28.57)$

7 (20.00)

3

$9(25.71)$

4

$1(2.86)$

Poor quality image

5 (14.29)

\section{Week 14}

1

$10(28.57)$

2

$8(22.86)$

3

$5(14.29)$

4

$4(11.43)$

5

$3(8.57)$

Poor quality image

5 (14.29) 


\section{Volume change}

\section{Week 9}

$<80 \%$

$20(57.14)$

$\geq 80 \%$

$10(28.57)$

Poor quality image

$5(14.29)$

\section{Week 14}

$<80 \%$

$10(28.57)$

$\geq 80 \%$

$20(57.14)$

Poor quality image

$5(14.29)$

\section{Postoperative pathological T-stage(ypT)}

T0

$8(22.86)$

$\mathrm{T} 1$

$2(5.71)$

$\mathrm{T} 2$

$7(20.00)$

T3

$3(8.57)$

T3a

$6(17.14)$

$\mathrm{T} 3 \mathrm{~b}$

$2(5.71)$

$\mathrm{T} 4 \mathrm{a}$

$1(2.86)$

$\mathrm{T} 4 \mathrm{~b}$

$4(11.43)$

Missing

$2(5.71)$

Postoperative pathological tumour regression grading (ypTRG)

0

1

$3(8.57)$

$8(22.86)$

2

$6(17.14)$

3

$8(22.86)$

4

Missing

$* \mathrm{~N}=35$ patients; values $\mathrm{n}(\%)$.

NACRT - neoadjuvant chemoradiotherapy 
Table 3 - Results from logistic regression analysis with pathological T-stage (ypT) as an outcome variable

\begin{tabular}{|c|c|c|c|c|c|c|}
\hline \multirow[t]{2}{*}{ Variable } & \multicolumn{2}{|c|}{ Pathology Outcome } & \multirow[t]{2}{*}{$\mathbf{O R}^{\#}$} & \multirow[t]{2}{*}{$95 \% \mathrm{CI}^{\#}$} & \multirow{2}{*}{$\begin{array}{c}\text { p- } \\
\text { value }^{\#}\end{array}$} & \multirow[t]{2}{*}{ p $_{\text {FET }} *$} \\
\hline & Favourable & Unfavourable & & & & \\
\hline Age (years) mean (SD) & $65.5(10.8)$ & $68(8.8)$ & 1.0 & $0.9,1.1$ & 0.56 & \\
\hline \multicolumn{7}{|l|}{ Sex } \\
\hline Male & 20 & 6 & 1.8 & $0.2,18.0$ & 0.62 & \\
\hline Female & 6 & 1 & & & & \\
\hline \multicolumn{7}{|l|}{ Week 9 ymrT-stage } \\
\hline Favourable & 16 & 0 & N/A & N/A & N/A & 0.002 \\
\hline Unfavourable & 6 & 6 & & & & \\
\hline \multicolumn{7}{|l|}{ Week 14 ymrT-stage } \\
\hline Favourable & 19 & 1 & 31.7 & $2.7,373.7$ & 0.006 & 0.003 \\
\hline Unfavourable & 3 & 5 & & & & \\
\hline \multicolumn{7}{|l|}{ Week 9 ymrTRG } \\
\hline Favourable & 17 & 2 & $6.8^{\wedge}$ & $0.9,48.7$ & 0.056 & 0.064 \\
\hline Unfavourable & 5 & 4 & & & & \\
\hline \multicolumn{7}{|l|}{ Week 14 ymrTRG } \\
\hline Favourable & 20 & 2 & 20.0 & $2.1,186.9$ & 0.009 & 0.01 \\
\hline Unfavourable & 2 & 4 & & & & \\
\hline \multicolumn{7}{|l|}{ Week 9 volume change } \\
\hline Favourable & 10 & 0 & N/A & N/A & N/A & 0.062 \\
\hline Unfavourable & 12 & 6 & & & & \\
\hline \multicolumn{7}{|l|}{ Week 14 volume change } \\
\hline Favourable & 18 & 4 & 22.5 & $2.0,249.2$ & 0.011 & 0.007 \\
\hline Unfavourable & 1 & 5 & & & & \\
\hline Adjusted for baseline & & & 16.7 & $1.4,197.1$ & $\mathbf{0 . 0 2 5}$ & \\
\hline
\end{tabular}

\# All OR, 95\%CI and p-values are unadjusted unless specified otherwise.

'Unable to adjust as this is the baseline value

$\mathrm{p}_{\mathrm{FET}} *$ Fishers Exact test

ymrT - MRI defined tumour T-stage post neoadjuvant chemoradiotherapy; ymrTRG - MRI

defined tumour regression grading post neoadjuvant chemoradiotherapy 
Table 4 - Logistic regression with pathological tumour regression grading (ypTRG) as the outcome variable

\begin{tabular}{|c|c|c|c|c|c|c|}
\hline \multirow[t]{2}{*}{ Variable } & \multicolumn{2}{|c|}{ Pathology Outcome } & \multirow[t]{2}{*}{$\mathbf{O R}^{\#}$} & \multirow[t]{2}{*}{$95 \% \mathrm{CI}^{\#}$} & \multirow{2}{*}{$\begin{array}{c}\text { p- } \\
\text { value }^{\#}\end{array}$} & \multirow[t]{2}{*}{$\mathbf{p}_{\mathrm{FET}}{ }^{*}$} \\
\hline & Favourable & Unfavourable & & & & \\
\hline Age years) mean (SD) & $63.9(45,88)$ & $68.1(56,83)$ & 1.0 & $0.9,1.0$ & 0.244 & \\
\hline \multicolumn{7}{|l|}{ Sex } \\
\hline Male & 10 & 16 & 9.6 & $1.0,92.0$ & 0.052 & \\
\hline Female & 6 & 1 & & & & \\
\hline \multicolumn{7}{|l|}{ Week 9 ymrT-stage } \\
\hline Favourable & 12 & 4 & 9.0 & $1.6,50.7$ & 0.013 & 0.020 \\
\hline Unfavourable & 3 & 9 & & & & \\
\hline Adjusted for baseline & & & 3.4 & $0.7,16.2$ & 0.128 & \\
\hline \multicolumn{7}{|l|}{ Week 14 ymrT-stage } \\
\hline Favourable & 14 & 6 & 16.3 & $1.6,163.4$ & 0.017 & 0.011 \\
\hline Unfavourable & 1 & 7 & & & & \\
\hline Adjusted for baseline & & & 17.5 & $1.2,250.4$ & 0.035 & \\
\hline \multicolumn{7}{|l|}{ Week 9 ymrTRG } \\
\hline Favourable & 13 & 6 & $7.6^{\wedge}$ & $1.2,48.0$ & 0.031 & 0.042 \\
\hline Unfavourable & 2 & 7 & & & & \\
\hline \multicolumn{7}{|l|}{ Week 14 ymrTRG } \\
\hline Favourable & 15 & 7 & N/A & N/A & N/A & 0.005 \\
\hline Unfavourable & 0 & 6 & & & & \\
\hline \multicolumn{7}{|l|}{$\begin{array}{l}\text { Week } 9 \text { volume } \\
\text { change }\end{array}$} \\
\hline Favourable & 8 & 2 & 6.3 & $1.0,38.7$ & 0.047 & 0.055 \\
\hline Unfavourable & 7 & 11 & & & & \\
\hline Adjusted for baseline & & & 5.1 & $0.8,32.6$ & 0.085 & \\
\hline \multicolumn{7}{|l|}{$\begin{array}{l}\text { Week } 14 \text { volume } \\
\text { change }\end{array}$} \\
\hline Favourable & 14 & 5 & 22.4 & $2.2,227$ & 0.009 & 0.004 \\
\hline Unfavourable & 1 & 8 & & & & \\
\hline Adjusted for baseline & & & 18.7 & $1.8,198.9$ & 0.015 & \\
\hline
\end{tabular}

${ }^{\wedge}$ Unable to adjust as this is the baseline value

${ }^{\#}$ All OR, 95\% CI and p-values are unadjusted unless specified otherwise. 
$\mathrm{p}_{\mathrm{FET}} *$ Fishers Exact test

ymrT - MRI defined tumour T-stage post neoadjuvant chemoradiotherapy; ymrTRG - MRI defined tumour regression grading post neoadjuvant chemoradiotherapy 
Figure 1: Sensitivity and specificity (\%) for predictor variables: MRI defined post-neoadjuvant chemoradiotherapy T-stage (ymrT), MRI defined post-neoadjuvant chemoradiotherapy tumour regression grading (ymrTRG) and volume change at week 9 and week 14 as derived from the logistic regression models with pathological defined T-stage (ypT) and pathological defined tumour regression grading (ypTRG) as outcome variables. The area under the receiver operating curve (ROC), together with its $95 \% \mathrm{CI}$, are given in Appendix 2 for each of the predictors. 


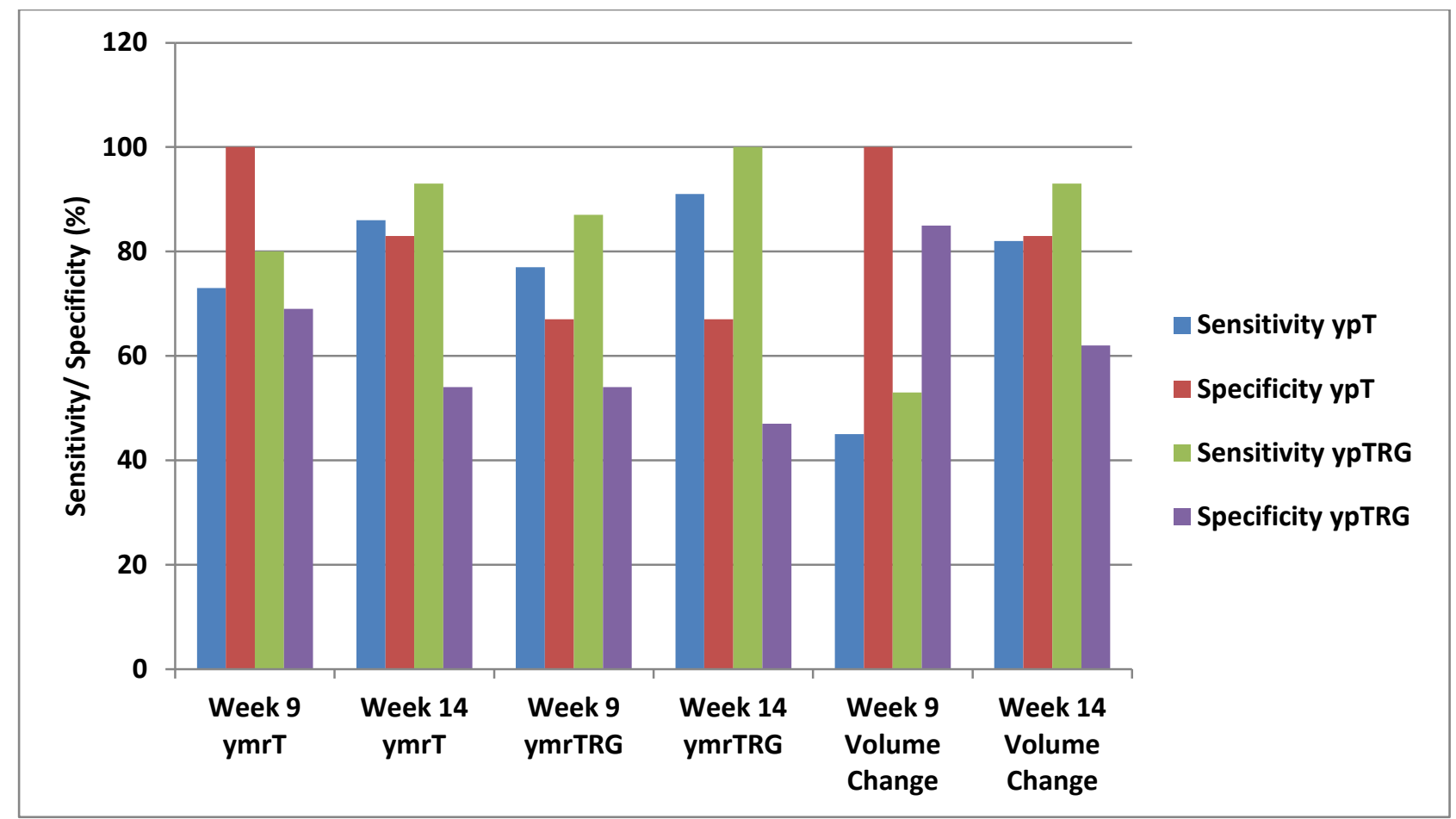


Supplementary files
Click here to download Supplementary files: Appendix 1-online only.docx

Supplementary files
Click here to download Supplementary files: Appendix 1-online only.docx

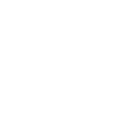

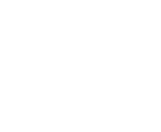

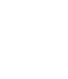

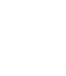

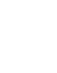
(

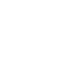
. . .

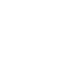

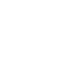

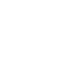

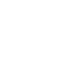

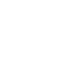

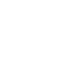

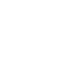

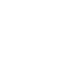

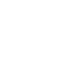

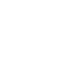

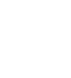

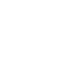

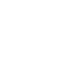

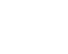


Click here to download Supplementary files: Appendix 2-online only.docx Supplementary files
Click here to downlo

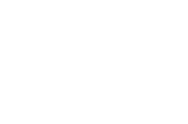

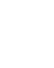
(1)

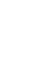
(1) (1) (1)

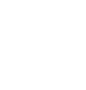
(1) (1) . . . . . . . . . . . . . . . 


\section{Conflict of Interest}

$M P W G$ - has received research grants from: National Institute of Health Research, Medical Research Council, National Institute of Academic Anaesthesia (British Oxygen Company Chair of the Royal College of Anaesthetists), and the British Lung Foundation. He leads the Xtreme-Everest Oxygen Research Consortium, which has received un-restricted research grant funding from: BOC Medical (Linde Group) ElyLilly Critical Care, Smiths Medical, Deltex Medical, London Clinic, Rolex.

GB - Supported by the Royal Marsden Cancer Charity and the NIHR Biomedical Research Centre

All other authors have no declarations 